Fecha de recepción: febrero 2017 Fecha de aceptación: agosto 2017 Versión final: septiembre 2018

\section{Modeling design conversation}

Celso Carnos Scaletsky ${ }^{\star}$, Chiara Del Gaudio ${ }^{\star 2}$, Filipe Campelo Xavier da Costa ${ }^{\star 3}$, Gerry Derksen ${ }^{\star 4}$, Guilherme Corrêa Meyer ${ }^{\star 5}$, Juan de la Rosa ${ }^{\star 6}$, Piotr Michura ${ }^{\star 7}$ y Stan Ruecker ${ }^{\star 8}$

Resumen: En este artículo, se presenta el desarrollo experimental del sistema analítico físico 3D para la realización de análisis cualitativos y cómo ha contribuido al proceso de reflexión ente los miembros del equipo de diseño. El objetivo principal de este proyecto ha sido incrementar el involucramiento de los diseñadores en los análisis colaborativos y mejorar la calidad de sus resultados. Este texto se ha focalizado en entender el rol de la conversación en la consecución de los dos objetivos.

En total, se han realizado una serie de 14 workshops. Aquí se presenta en detalle, como caso de estudio, sólo una de las sesiones, seleccionada debido a que posee un estándar bastante sofisticado en relación a que los participantes rechazaron la brevedad analítica para producir, en cambio, una compleja meta-interpretación de la información.

Palabras clave: análisis cualitativo - proceso de diseño - modelo convencional - interpretación colaborativa - hermenéutica.

[Resúmenes en inglés y portugués en la página 51]

${ }^{*}$ Professor of the Post Graduate Program in Design at University of Vale do Rio dos Sinos - UNISINOS, in Porto Alegre, Brazil. His research is oriented to Project Theory with a specialization in Strategic Design.

${ }^{\left({ }^{2}\right)}$ Post-doctoral researcher in Design at University of Vale do Rio dos Sinos, Brazil. Her research interests are in participatory design, social innovation, and in the designer's action and integration in conflict-affected and fragile urban areas.

${ }^{*}{ }^{* 3)}$ Professor and coordinator of Design Graduate Program at University of Vale do Rio dos Sinos - UNISINOS, Porto Alegre, Brazil. His research interests are related to user experience studies and design for emotion perspective in design research.

${ }^{\left({ }^{*}\right)}$ Professor at Winthrop University in South Carolina, USA, and coordinator of the Interactive Media program in the Department of Digital Information Design. His areas of research include usability and heuristic evaluation, experience design, and natural language processing. 
$\left.{ }^{*}{ }^{*}\right)$ Professor of the Post Graduate Program in Design at University of Vale do Rio dos Sinos - UNISINOS, in Porto Alegre, Brazil. His research includes the use of prototype to build knowledge in design.

${ }^{\left({ }^{*}\right)}$ Associate professor at Universidad Nacional de Colombia’s School of Graphic Design. His current research focuses on behavioral transformation on communities through the use of design.

${ }^{\left({ }^{*}\right)} \mathrm{PhD}$. Lecturer at the Department of Design Fundamentals, Faculty of Industrial Design, Academy of Fine Arts in Krakow, Poland. His current research interests focus on conversation modeling theory and practice in interaction design fostering agency and engagement of stakeholders and generative potential of contextual factors.

${ }^{\left({ }^{*}\right)} \mathrm{PhD}$. Associate Professor at the IIT Institute of Design, an Adjunct Professor at the University of Alberta's Humanities Computing Program, and in the University of Victoria's Faculty of Humanities. His current research interests in the areas of humanities visualization, the future of reading, and information design. His work to date has focused on developing prototypes to support the hermeneutic or interpretive process.

\section{Introducción}

Diseñar requiere conocimientos de construcción y, en el proceso, varios actores se ven involucrados. Éstos argumentan sobre su proyecto, discuten entre ellos mismos, y comunican a los actores externos. Aunque el modelo conversacional ha sido puesto en práctica por un sinnúmero de disciplinas por muchos años (Stevers y Sydney, 2014), la naturaleza exacta de la conversación y sus beneficios aún permanecen sin ser extendidas a los miembros del equipo de diseño.

Cuando la conversación es mencionada con respecto al diseño, es asiduamente presentada en términos del proceso reflexivo que la práctica requiere, involucrando este tipo de conversación con el diseño mismo, tal como lo sostiene Schön (1987).

La conversación es un proceso de confrontación de ideas, de negociación, práctica y error, y persuasión, ya sea en la búsqueda de consenso o asumiendo diversos y contradictorios puntos de vista. ¿Cómo se llevan adelante las conversaciones en el proceso de diseño? ¿Es posible diseñar un artefacto mediador que brinde, a este tipo de proceso conversacional, algún tipo de ayuda para ser más colaborativo y generador de resultados más sofisticados? Partiendo de la investigación desde una perspectiva de diseño, nos hemos focalizado en comprender cómo un sistema experimental físico 3D para el análisis de transcripciones puede ser usado por un equipo para representar o desarrollar un nuevo conocimiento durante el proceso de diseño.

El modelo resultante no es un artefacto en el sentido de un diseño físico; es un modelo de análisis de trascripción de entrevistas llevado adelante por el equipo de diseñadores, $\mathrm{y}$-en tanto artefacto- podría ser considerado un artefacto de análisis. Este sistema es usado por 
el equipo de diseño como un mediador para la representación e interpretación de contenido referido a la conversación sobre diseño. Partiendo de la suposición que la combinación de ideas y conocimientos presentes en cada conversación de diseño son parte del proceso de diseño mismo, compartir las reflexiones se torna una práctica significativa para los profesionales de la disciplina.

El modelo físico 3D, como mediador, debería representar visual, espacial y temporalmente los fenómenos articulados en la conversación. Los recursos visuales y espaciales son usualmente mal calificados como una forma primitiva de pensamiento, y como una manera pre-verbal y no-lógica de razonamiento (Goldshimidt, 1994). En el proceso de diseño, de todas maneras, la apertura y la lógica no línea del pensamiento visual contribuyen combinando miradas a menudo antagónicas.

Estas características también contribuyen a la generación de ideas y conceptos. El modelo físico 3D también involucra la forma en que los diseñadores usan su tiempo, como -por ejemplo- los recursos de diseño que raramente son una secuencia bien estructurada de pasos. Los diseñadores frecuentemente usan las representaciones visuales, espaciales y temporales para convertir las ideas abstractas en ideas tangibles.

El modelo propuesto en esta investigación tiene un doble objetivo. Por un lado, como ya fue mencionado, se pretende investigar como el modelo físico 3D puede ser un mecanismos de representación, interpretación y constricción de nuevos contenidos, de nuevas ideas que emergen durante la etapa de análisis del procesos de diseño. Por otra parte, se busca comprender como el uso de este modelo puede conducir el desarrollo de nuevas teorías a partir de la conversación en el proceso de diseño.

En relación con el segundo objetivo, Legay (1996, pp. 45-46) sostiene que, en las ciencias, hay dos formas de construir un modelo. La primera parte de una teoría, y de esta forma el modelo es formulado por diferentes situaciones. El objetivo es fortalecer o debilitar una hipótesis. La segunda forma se basa en una situación dada sobre la que el modelo es construido, y a partir de éste se podría generar una teoría. Esta investigación es más cercana a esta segunda perspectiva. A partir de las conversaciones surgidas (como pueden ser las transcripciones de una entrevista), los modelos analíticos están formulados para representar, interpretar y construir nuevas narrativas. La observación de este proceso puede ayudar a formular teoría sobre las conversaciones de diseño. Morrison and Morgan (2008, p. 10), reflexionan sobre los modelos concebidos como instrumentos mediadores en ciencia, cuando proponen que:

...es común pensar esos modelos pueden ser derribados completamente por una teoría o una información. De todas maneras, si miramos de cerca la forma en que los modelos han sido construidos, podemos comenzar a ver las fuentes de su independencia. Justamente porque no son ni una cosa ni la otra, no son solo teoría o información -aunque estén típicamente involucrados con alguna de ellas (y generalmente con otros elementos "foráneos) - es que pueden mediar entre la teoría y el mundo.

En algunas de nuestras últimas investigaciones, hemos podido observar que tanto la construcción del modelo conversacional (hecho por diseñadores) como así también la inter- 
pretación o representación de contenidos son susceptibles a múltiples influencias. Todo objeto diseñado puede ser visto como una comunicación entre diseñadores y otros actores involucrados en el diseño. En el caso de la escultura conversacional, es decir, el modelo 3D propuesto, los roles de diversas personas podrían cambiar e influir en los resultados. Se pueden producir sorpresas o ideas inesperadas en un camino exploratorio trabajando en el espacio físico o mirando en una forma no lineal.

Puede caracterizarse este modelo como una surte de prototipo de ideas conexas. Estas vinculaciones, son asimismo, una manera de generar alternativas de diseño. Los diseñadores solían desarrollar prototipos o modelos para generar y testear ideas de diseño. El modelo conversacional permite abrir un acercamiento a la comprensión, dejando espacio para la representación y la exploración de lo inesperado, para proveer rápidos "prototipos" de conexiones entre ideas, y para encontrar la "geometría" de las ideas entre el espacio de las alternativas (Frazer, 1995).

Para Møller y Tollestrup, los artefactos físicos habilitan y estimulan conversaciones entre diseñadores, usuarios y stakeholders por medio de "Conceptos Experimentales Personales". Este acercamiento permite "darle un significado a lo relacionado con su proyecto o su vida cotidiana, y los habilita para hacer explícito su laxo conocimiento" (p. 106). Sumado a esto, los artefactos físicos les dan lugar para crear un segundo nivel de comprensión, que promueve discusiones y negociaciones, típicas de cualquier conversación.

Piaget (1954) y Vygotsky (1986), en busca de comprender los eventos cognitivos ocultos en una situación problemática dada, hicieron una serie de experimentos con artefactos físicos. En sus experimentaciones, los participantes eran alentados a vincularse con los objetos mediante explicaciones verbales o manipulaciones. Especialmente en el caso de los niños, se subrayó la posibilidad de manipular el objeto real como un elemento crucial en toda la situación experimental, que lo ayudó a concretar sus operaciones y evitar problemas al proporcionar explicaciones verbales.

Star y Griesemer (1989) introdujeron el concepto de objeto-frontera, que colabora con conversaciones mediadas; en este sentido, el sistema físico 3D que hemos creado puede ser entendido como una suerte de objeto-frontera. De todas formas, esto difiere en algunos puntos de las cuatro categorías que los autores sostienen como las más comunes: repositorios, tipos ideales, fronteras coincidentes y formas estandarizadas. En cambio, este objetofrontera o artefacto de mediación actúa como un rotafolio o una frontera blanca: no es un objeto que ya exista como representación de una idea; por el contrario, es una tabula rasa a través de la cual las ideas pueden ser colaborativamente desarrolladas y compartidas. Para comprender mejor el sistema, tal como lo describen Johansson y Arvola (2007) como una estructuración de recursos, con la que quieren denominar a un objeto, método o sistema que provee un andamio de pensamiento, infienciando no solo qué se piensa sino también qué opciones están disponibles.

\section{Método}

Con el fin de arrojar algo de luz sobre esta temática, hemos desarrollado una serie de prototipos funcionales del sistema físico 3D de análisis para facilitar la conversación y la 
producción de conocimiento en el proceso de diseño. El objetivo del sistema fue permitir a nuestros participantes crear un modelo 3D de su comprensión de texto. Luego, se llevó adelante una serie de 14 workshops (de dos horas), con una variación entre 2 a 7 participantes por cada sesión.

Con el propósito de focalizar cercanamente en las interacciones y resultados de la sesión, se eligió una de ellas que estuvo compuesta por un grupo de estudiantes de doctorado (participantes 1, 2, 3, 4 y 5). Se les entregó un texto y se les solicitó construir un modelo relacional espacial de los contenidos y, también comprender cómo el modelo/prototipo podía ser utilizado para aumentar la comprensión y las discusiones sobre el texto.

Más específicamente, el texto provisto contiene un diálogo en el que cuatro diseñadores hablan acerca de la construcción de escenarios de diseño para un nuevo sistema de información de una compañía líder global de diseño de mobiliario de oficina.

El modeling system consiste en un set de paneles separados que pueden ser ensamblados para construir grandes estructuras espaciales. Las estructuras resultantes permiten a los participantes posicionar diferentes tipos de información en un espacio 3D. Los paneles estaban fabricados en acrílico (un poliéster transparente). De este modo fue posible escribir y pegar notas, dibujos y trabajos -que los participantes del debate pudieron utilizar para argumentar la estructura- de ambos lados de los paneles. La fantástica ventaja de este tipo particular de modelo de diseño fue que los elementos pudieron ser libremente usados para crear una arquitectura a cielo abierto.

El workshop grabado y, luego, examinado de acuerdo a la técnica de análisis de contenidos de Bardin (1991). Las categorías utilizadas para el análisis de contenido fueron: 1) interpretación del texto, 2) el proceso de relación del contenido con el modelo/prototipo, 3) la representación de los contenidos del sistema de narrativas, y 4) la interacción física entre los participantes y el modelo. El protocolo de análisis es presentado en el siguiente apartado.

\section{Observación del proceso de conversasional}

El análisis del video grabado en el workshop permitió recolectar información sobre cuatro categorías. Se han desarrollado parámetros en torno a las implicaciones del uso del modelo para la representación o desarrollo de nuevos conocimientos durante el debate. La información más relevante de cada categoría se presenta a continuación.

\subsection{Interpretación del texto}

En lo que respecta a como los participantes interpretaron la transcripción, se identificaron dinámicas significativas en el proceso de interpretación, y algunos aspectos clave en la relación con la actividad sugerida. Tal como se explicó previamente, se les brindó a los participantes dos horas, el texto y el modelo. En este esquema, se observó que:

- Los participantes invirtieron la mitad del tiempo en debatir el contenido, pero casi sin abordar el modelo. Esta parte de la conversación sucedió sobretodo verbalmente. Sólo 
hacia el final de este lapso de tiempo ellos comenzaron a interactuar con el modelo, pero de un modo limitado.

- La interacción con los contenidos fue realzada en relación con el modelo. De hecho, en el debate los participantes se preguntaron a sí mismos qué era importante para el modelo. Esto surgió en repetidos momentos durante todo el proceso. Por ejemplo, uno de los participante dijo: "en vez de ayudarnos a analizar, el modelo nos está ayudando a entender qué analizar y cómo analizarlo" (p1, 01:07:50).

- Aún cuando ellos se preguntaron qué contenidos eran importantes para el modelo, la selección inicial de aquellos que consideraban relevantes fue realizada sin considerar la interacción con el modelo y analizado el texto de un modo tradicional. Individualmente y luego como actividad grupal, leyeron el texto nuevamente en busca de identificar las unidades de análisis, las marcaron y las escribieron en unos post-its.

- Una vez avanzado el debate, comenzaron a reflexionar sobre las diferentes interpretaciones del contenido y sobre la relevancia de seleccionarlo y colocarlo en un esquema de representación. Esto surgió cuando se inició una interacción mayor con el modelo: el proceso fomentó una discusión donde las ideas de todos los participantes fueron consideradas, intercambiadas y comparadas.

- En muchos momentos notaron y discutieron sobre como, al utilizar el modelo para la interpretación, los había ayudado a clarificar las ideas y a entender los puntos conflictivos desde una perspectiva particular de análisis del texto.

De acuerdo con estos resultados, algunos aspectos clave ha emergido: la resistencia inicia de los participantes hacia una forma diversa de pensamiento y de análisis que el modelo permitía y sugería; la evolución de la interpretación del contenido cuando ellos comenzaron a usar el modelo y el crecimiento de su comprensión y debate; cómo el modelo propició la comparación y la puesta en común de las ideas; y, finalmente, que la interpretación a través del modelo les permitió rápidamente identificar los puntos difíciles de comprender.

\subsection{El proceso de relaciones en el modelo de contenidos}

El proceso sobre cómo los participantes relacionaron el contenido y el modelo/prototipo incluye dos procesos de comprensión: cómo ellos procedieron y cómo ellos representaron:

- Para todos ellos, durante la primera hora, no fue claro como debían usar el modelo y cómo debían relacionado el contenido a él.

- Los participantes realizaron varios intentos antes de asentar la versión final de relación de contenidos y modelo. Al principio, quizás por el protocolo sugerido al inicio de la sesión, intentaron redefinir la manera de representación sin interactuar físicamente con el modelo, y sin una relación real como el contenido y las posibilidades de representación y comunicación del modelo. Por ejemplo, debatieron sobre tres estructuras alternativas: cronológica, interacción entre speakers, clusters de ideas. Las alternativas no fueron generadas usando el modelo como medio y objeto de debate; sino, en cambio, considerando las experiencias previas de los participantes. Otro ejemplo de falta de conexión entre el con- 
tenido y el modelo en el debate inicia de representación fue que se comenzó seleccionado tópicos clave en un forma estándar, colocando notas en post-it sobre una superficie plana (es decir, en paneles del modelo colocados uno al lado del otro).

- La percepción de los participantes sobre como usar el modelo al comienzo del proceso de definición de los protocolos, los llevó a discutir el objetivo del taller y de la investigación. - La dimensión colectiva del debate y del proceso de vinculación del contenido con el modelo, creció junto con la interacción de los participantes con el modelo. Las sugerencias diferentes y las discusiones colectivas señalaron que un proceso de construcción colectiva de conocimiento ocurrió.

- La comprensión del uso del modelo creció rápidamente cuando los participantes adoptaron una "actitud divertida" y se permitieron momentos de experimentación.

- A través de la experimentación ellos identificaron muchas posibilidades de representación por medio del modelo: posiciones con respecto a las miradas, planta o nivel, visiones desde adentro o desde afuera.

- Dos procesos diferentes suceden al mismo tiempo a lo largo del workshop: el proceso de vinculación del contenido con el modelo, y el proceso de exploración sobre cómo el modelo permitió a los participantes al mismo tiempo representar y desarrollar una idea. Esto puede ser ejemplificado con el comentario de uno de los participantes: "hay múltiples cosas que pueden estar en un panel. Esto es una realidad que es diferente de una comprensión" (p.2, 59:09). En ese momento, no estaban debatiendo sobre la representación del contenido, pero sí de las posibilidades de representación que el modelo ofrecía. Esta mezcla de procesos no fue siempre simple de gestionar para los participantes; muchas veces condujo a la confusión de unos con otros.

- El proceso de comprensión sobre cómo utilizar el modelo involucró una intensa experimentación que duró una hora y media. En los últimos 30 minutos, el grupo acertó en definir el protocolo para el uso y representación de contenido en el modelo.

- Sin embargo, los participantes no representaron realmente el contenido con el modelo, sólo teorizaron sobre cómo hacerlo: a través de las interacciones analizaron varias posibilidades de representación, pero la real no tuvo lugar. Por ejemplo, luego de una hora, debatieron sobre la posibilidad de plantear una vista interior (para cada participante) y una vista exterior (para el consenso del grupo). Aunque la estructura fue construida, sólo se agregaron post-it preexistentes como contenido.

De este modo, es posible observar la inicial disconformidad y el esfuerzo realizado para cambiar la manera institucionalizada de pensar, analizar y representar; el proceso de construcción del conocimiento tiene una importa empírica y táctil; la relevancia de los momentos distendidos; el fomento de la dimensión colectiva; el gap existente entre la consigna de representación y los resultados finales; la superposición de dos procesos diferentes -la comprensión del potencial del modelo y de la representación de contenidos y, finalmente, las diferentes cualidades brindadas por el modelo-. 


\subsection{La representación del contendido del texto en el modelo/prototipo}

Se recolectó una serie de información acerca de cómo el modelo fue usado para representar el contenido del texto brindado. En lo que a esto respecta, es necesario observar que los participantes establecieron un criterio de representación que les permitía tanto estructurar jerarquías y relaciones entre la información, como plantear diferentes perspectivas. Por medio de un debate, decidieron que era importante mostrar los diferentes individuos y sus diferentes puntos de vista. El modo en que decidieron plasmar el contenido narrativo en el modelo, es el siguiente:

- Una base sobre la que representar los análisis individuales.

- Una segunda capa que represente los puntos irresueltos.

- Otra capa que muestre los asuntos resueltos.

- En la parte superior de la pirámide, los conocimientos e ideas.

- Para mostrar las adjetivaciones (por ejemplo, acuerdo o desacuerdo) se utilizaron colores.

Los participantes notaron que el protocolo que sugirieron podía ser útil tanto para representar el contenido del texto como modelo de organización de la compañía, con las perspectivas de las diferentes partes.

Finalmente, los participantes realizaron algo más que representar los puntos principales del texto: investigaron cómo el modelo puede ayudar a las discusiones sobre contenidos y, algunas veces, estimular las dinámicas de comunicación dentro de una organización.

\subsection{La interacción física entre los participantes y el modelo}

La naturaleza física y material del modelo es uno de los aspectos clave de la investigación. Por este motivo es relevante observar la dinámica de la interacción y su relación con la comprensión y el desarrollo del conocimiento. El análisis del video arrojó que:

- Al inicio los participantes no tocaban el modelo. Fue sentados alrededor de una mesa donde debatieron sobre el contenido.

- Luego, comenzaron a usar la estructura de ejemplos creada por los organizadores del workshop, aún cuando no existía una razón particular para mantenerla.

- La interacción física real con el modelo comenzó lentamente. Por ejemplo, después de que un participante agregase un post-it con una nota sobre la estructura de ejemplo, otro de ellos cambió su posición para poder ver mejor qué había añadido su compañero. Las primeras interacciones fueron realizadas por los más relajados y extrovertidos del grupo.

- La interacción creció en intensidad luego de que algunos miembros del grupo usasen los paneles en una manera divertida para explicar y argumentar su posición. Por ejemplo, uno de los participantes explicó cómo es posible "ver" el texto "a través" del modelo haciendo un cubo y sosteniéndolo sobre su rostro como si fuesen gafas.

- Después de desmontar la estructura de ejemplo y de proponer el primer criterio, los participantes empezaron a usar los elementos físicos en una forma más familiar. 
- Más adelante en el proceso, el razonamiento cambió de dirección. Este era, originalmente, sobre ideas teóricas acerca de cómo usar el modelo; y se modificó comenzado desde el modelo a explorar las posibilidades. Tal como lo sostuvo uno de los participantes: "sólo construyámoslo y veamos qué pasa" (p.1; 01:08:00).

- Luego de que el modelo se volviese más cercano, comenzaron a utilizarlo para explicar las ideas propias a los demás.

Esta última categoría claramente muestra tanto la relación directa entre el razonamiento y la interacción física con el modelo; como el tiempo necesario para familiarizarse con su uso y la gradualidad de este proceso. Al mismo tiempo, se puedo observar como la interacción cambió la dirección del proceso de razonamiento tanto que al final fue utilizado para explicar y sostener argumentos. El modelo permitió tanto asistir en la expresión de ideas como testearlas.

\section{Discusión sobre el uso del modelo}

El proceso de interpretación de un texto utilizando el modelo físico parece ser diferente de uno regular (en el que se recurren a plataformas tradicionales como un pizarrón o un anotador, por ejemplo). Esta distinción puede ser relacionada con el tipo de actividad que el modelo requiere, como intuición, creatividad, elaboración, testeo. Cuando los participantes necesitan pensar sobre cuál es la mejor manera de usar los paneles para elaborar estructuras adecuadas para organizar contenidos, comienzan a razonar diferente. Este tipo de modelos también comprenden apertura, vulnerabilidad y abducción colaborativa.

A pesar de que el sistema de modelo experimental usado para este estudio tiene algunas limitaciones físicas (en lo referido a los materiales, formas, conexiones), permite también la flexibilidad y estimula la producción. Es esencialmente generador. Puede ser visto como una actividad de diseño en sí misma, espacialmente en lo que respecta a los componentes reflexivos (Schön, 1987). Para elegir como combinar los paneles se requieren decisiones sobre la forma de la estructura (uno puede construir un simple cubo, mientras otros pueden desarrollar formas complejas). Este tipo de decisión, que emerge durante el proceso, se vincula a cómo se usa el modelo y no a cuál es el contenido del texto. Asumiendo el desafío de elaborar una buena estructura que pueda recibir la interpretación del contenido, los participantes de alguna manera abandonar la reflexión sobre el texto (tal como ya se dijo, el modelo presentó muy poca información explícita sobre la transcripción de la entrevista). Esta situación puede estar relacionada con el background de los participantes y su proximidad con formas de razonamiento del diseño.

Es interesante resaltar que el modelo, de algún modo, modificó la forma en que el contenido es interpretado y viceversa. La tarea general presentada en el workshop solicitaba a los participantes que construyesen una estructura (usando los paneles acrílicos suministrados) para organizar el contenido de una conversación específica (una entrevista transcripta). A pesar de que esta indicación general sugería que el modelo debía estar orientado a los contenidos, el proceso reveló una dinámica diferente. Tal como se describió previamente, los participantes decidieron comenzar leyendo la entrevista y discutiendo 
el contenido. Sin embargo, durante el desarrollo del workshop la forma de razonamiento cambió de dirección. Ya no estuvo más dirigida desde el contenido de la entrevista hacia el modelo, sino que partiendo desde el modelo se comenzaron a explorar las posibilidades (así como lo remarcó el participante1; 01:08:00). De esta forma, se identificó en el proceso una suerte de dinámicas recursivas: desde el contenido al modelo y desde el modelo al contenido, respectivamente.

El proceso de usar el modelo para la representación de contenido puede inductivamente generar nuevo conocimiento. En este workoshop en particular se partió de una situación específica y se trabajó sobre su interpretación. El razonamiento del grupo se expresó a través de un concepto potencial. Uno de ellos consideró que podía proveerles una comprensión teórica del asunto. Esta generalización necesita una investigación particular sobre cómo el concepto funcionaría en diferentes situaciones, verificando y amplificando los resultados, deductivamente. Este mecanismo puede ser respaldado por la afirmación de Legay (1996) sobre cómo construir nuevas teorías.

Tal como ya se describió, el contenido específico de la entrevista nueva fue completamente plasmado en el modelo, en los paneles. El modelo fue construido para intentar visibilizar el concepto potencial que los participantes identificaron durante los debates colectivos. El protocolo que generaron a través de su interacción con el modelo es, de alguna manera, ese concepto potencial.

El modelo en su totalidad fue construido bajo la idea de que para analizar la entrevista era necesario considerar las perspectivas de los diferentes agentes involucrados: el intérprete, el entrevistador y los entrevistados. Se estableció un tipo de concepto relacional, suficiente para representar la compresión de los participantes sobre el proceso de interpretación. Este fue elaborado en todo el proceso, durante las interacciones con el contenido, el modelo y los participantes. Así el modelo modificó, de algún modo, su requerimiento contingente (como sugirió en tarea preliminar: una estructura para esa entrevista específica), asumiendo un aspecto más general, el de la perspectiva de usuarios múltiples que existe en las interpretaciones de las entrevistas. Por ende, la definición del protocolo fue obtenida inicialmente a través de un razonamiento deductivo, pero luego se modificó a un criterio nivel más amplio.

Es arduo detallar con precisión la evidencia de los momentos en los que los participantes formaron este concepto. El proceso abarcó diferentes aspectos, algunos de ellos difíciles de identificar. Pero algunos episodios progresivos puede ilustrar las dinámicas de interacción entre los participantes, el contenido y el modelo. El participante 1 demostró, usando los paneles (y construyendo un cubo), como se podía "ver" la conversación "a través" del modelo: "Pensaba que, en vez de estar ahí afuera, qué pasaría si estaba en mí, y si perspectiva fue calibrada por esta lente". El participante 3 notó que el modelo creado era insuficiente para representar todas las perspectivas: "el problema es que ahora se ha convertido solo en uno, en las ideas individuales, en lugar de en lo que queríamos compartir". El participante 4 sostuvo un panel con post.it en ambas caras, explicando la posibilidad de representar una visión interior (de cada interprete) y una exterior (que muestre el consenso del grupo). El participante 2 gestualmente indicó que cada uno debería tener un par de cubos, todos conectados de persona a persona. El participante 3 sostuvo "tal como hay diferentes personas, hay diferentes visiones". El participante 5 sugirió una localización "si tu visión coincide con 
al de los demás, entonces debería ir en la parte interior". El participante 1 propuso un modelo de visualización con 5 caras (una por cada interprete) que englobaría el modelo existente. Esta secuencia de eventos ilustra cómo las ideas parecerían influirse entre sí, así como el papel que desempeña la presencia del modelo en el recorrido de surgimiento de los argumentos.

Está claro que este proceso asumió una dimensión colectiva desde el momento en que los participantes comenzaron a interactuar más con el modelo. El debate (colectivo) sucedió apoyado por las actividades de construcción, cuando se utilizó el modelo para confrontar ideas, clarificar y mejorar las propuestas comunes. Tal como es posible observar, muchas veces los participantes usaron el modelo para reconocer, compartir y comprender sus argumentos. Esto parecería que se sucedió debido a las competencias múltiples que el modelo posee. En primer lugar permitió a los participantes presentar sus ideas, usándolo como plataforma de intermediación, que asistió en la expresión de las ideas. Al mismo tiempo, facilitó la evaluación de esas ideas, trabajando como un dispositivo de validación.

Pero el modelo también influenció (y sugirió) la interpretación, sumando elementos nuevos al debate. A través de este proceso de habilitación, testeo y persuasión, los participante identificaron y también refutaron muchas posibilidades de representación (comenzaron proponiendo un criterio general, tal como presentó Jihyun Sun, en su trabajo de PhD -no publicado- sobre el modelo ALP: espacio aplicado, espacio latente, espacio periférico y la finalmente la idea del sistema relacional de usuarios múltiples).

\section{Conclusiones}

En pocas palabras, considerando la discusión sobre los resultados recién presentados, fue posible identificar algunos puntos principales: 1) El proceso de interpretación de texto usando el modelo físico parecería ser diferente al regular, en parte porque los componentes generativos del modelo. 2) El modelo modificó la forma en que el contenido fue interpretado y viceversa: el modelo intermedió la interpretación, siendo un mecanismo de validación, y también influenció la comprensión. 3) El modelo puede ayudar a la construcción de conocimiento en el campo del diseño, siguiendo razonamientos inductivos. Esto podría verse reflejado en la forma de un concepto potencial que representa la comprensión colectiva de los participantes, desarrollada a lo largo de la actividad del workshop. 4) La dimensión colectiva apareció cuando los participantes incorporaron el modelo al proceso. Éste promovió compromisos y mejoras en la interpretación colectiva. 5) A pesar de que el modelo parece conducir a una interpretación general de proceso, permitiendo a los participantes encontrar un concepto potencial, por ejemplo, no los impulsa a representar el contenido específico presente en la transcripción de la entrevista.

\section{Investigación futura}

Es difícil remarcar con precisión las diferencias entre el uso de este modelo o de otros mecanismos (software, hardware) para la interpretación de conversaciones. Algunas ca- 
racterísticas generales concernientes al modelo fueron ya descriptas, pero una nueva investigación debería abordar estas diferencias.

En esta etapa preliminar de la investigación, hemos identificado algunas evidencias acerca de cómo un modelo de este tipo puede mejorar el proceso de interpretación. El estudio abordó preguntas tales como: ¿Cómo puede el modelo facilitar la cooperación, la interacción y la comunicación? ¿Cuál es el resultado de tales interacciones?; ¿El proceso facilita la reflexión individual? ¿Qué elementos de una herramienta espacio-visual son necesarios para representar conceptos?

En este estudio, los participantes fueron todos diseñadores e investigadores (con diferentes grados de expertise). Dedicaron, al menos, la mitad del tiempo del workshop a intentar generar alternativas, construir estructuras, manejar los paneles, explorar posibilidades, utilizando sus estrategias existentes como diseñadores. También era evidente que los participantes siempre estaban tratando de comprender el objetivo de la actividad. Esto pudo estar relacionado con el background en investigación científica que todos ellos tenían. Tal vez, un grupo con una formación podría funcionar de otra manera.

Como conclusión puede afirmarse que es importante llevar adelante más investigaciones sobre el uso de modelos físicos 3D para el proceso de interpretación. Este tipo de trabajos nos permitiría examinar tanto las particularidades de los contextos específicos como las cuestiones comunes.

\section{Referencias}

Bardin, L. (1991). L'analyse de contenu. Paris: PUF.

Frazer, J. (1995). An Evolutionary Architecture. Architectural Association.

Goldschmidt, G. (1994). On visual design thinking: the vis kids of architecture. Design Studies Vol $15 \mathrm{~N}^{\circ} 2$, Kidlington: Elsevier.

Johansson, M., \& Arvola, M. (2007). A Case Study of How User Interface Sketches, Scenarios and Computer Prototypes Structure Stakeholder Meetings.

Legay, J-M (1996). L'expérience et le modèle: Un discours sur la méthode. Paris: INRA Editions.

Møller, L. \& and Tollestrup, C. (2013). Creating Shared Understanding in Product Development Teams: 'How to Build the Beginning'. London: Springer.

Morgan, M. \& Morrison, M. (2008). Model as mediating instruments. In Models as Mediators: Perspective on Natural and Social Science. Cambridge: Cambridge University Press. Piaget, J. (1954). The Construction of Reality in the Child, Ballantine.

Sidnell, J. and Stivers, T., Eds. (2014). The Handbook of Conversation Analysis. Oxford: Wiley Blackwell.

Schön, D. (1987). Educating the Reflective Practioner Toward a New Design for Teaching and Learning in the Professions. San Francisco: Jossey-Bass, Publishers.

Star, S. L., \& Griesemer, J. R. (1989). Institutional ecology, 'translations' and boundary objects: Amateurs and professionals in Berkeley's Museum of Vertebrate Zoology. Social Studies of Science, 19.

Vygotsky, L. (1986). Thought and Language, MIT Press. 


\begin{abstract}
In this paper it is reported the development of an experimental 3D physical analytical system for doing qualitative analysis, and how we examined its use in supporting conversation among the members of a design team. Our larger goals in this project were to increase the engagement of designers in collaborative analysis, and to improve the quality of the outcomes. In this paper, we concentrate on trying to understand the role of conversation in reaching these two goals. In total, we have done a series of 14 workshops; here, we discuss in detail, as a case study, just one of the sessions. We chose it because it was quite sophisticated in the sense that the participants rejected the brief and yet produced a complex meta-interpretation of the data.
\end{abstract}

Keywords: qualitative analysis - design process - conversational model - collaboration interpretation - hermeneutics.

Resumo: Este artigo apresenta o desenvolvimento experimental de um sistema analítico físico 3D para a realização de análise qualitativa e como ele tem contribuído para o processo de reflexão entre os membros da equipe de design. O principal objetivo deste projeto tem sido aumentar o envolvimento de designers na análise colaborativo e melhorar a qualidade dos seus resultados. Este texto tem como foco a compreensão do papel da conversa na realização dos objetivos.

No total, houve uma série de 14 workshops. Aqui é apresentada em detalhe, como estudo de caso, apenas uma das sessões, selecionada porque ela tem um padrão bastante sofisticado: os participantes rejeitaram a brevidade analítica para produzir, em vez disso, uma meta-interpretação complexa de informação.

Palavras chave: análise qualitativa - design de processo - modelo convencional - representação colaborativa - hermenêutica. 